\title{
XIX.
}

Aus der chirurgischen Klinik in Greifswald (Prof. Helferich).

Ueber eine Geschwulst von schilddrüsenartigem Bau im Vemur.

\author{
Von \\ Dr. Carl Goebel, \\ Assistonzarzt der Klinik.
}

Im August 1897 wurde hierselbst eine Geschwulst von schilddrüsenartigem Bau im Femur beobachtet, deren histologische Untersuchung einige Besonderbeiten darbietet, und die auch klinisch von Interesse ist, da sie wobl ausser dem von $\mathrm{Ne} \mathrm{umann}{ }^{1}$ ) beschriebenen ähnlichen Fall der einzige bisher hier im Norden beobachtete sein durfte.

Krankengeschichte.

Frau A. aus Buttow in Hinterpommern, 54 Jahre alt, will früher stets gesund gewesen sein. Mehrere Lintbindungen sind gut verlaufen. Seit mehreren Jahren Menopause. Pat. ist vor $21 / 2$ Jahren gefallen und hat dabei den rechten Oberschenkel zwischen mittlerem und unterem Drittel gebrochen. Der Bruch heilte angeblich mit Verkürzung, so dass Pat. wieder gehen konnte. Doch erlitt sie 1/4 Jahr später von Neuem bei einem Fall auf dem Strassenpflaster einen Bruch an derselben Stelle. Seitdem liegt Pat. fortwährend, da die Fractur sich nicht consolidirte. Nur mit Krücken konnte sie sich herumbewegen, doch war dies selbst in letzter Zeit nicht mehr möglich. Wegen ihrer dadurch bedingten Hülflosigkeit, auch der Schmerzen halber wünscht Patientin Heilung durch Operation, eventuell Amputation.

Die Pat. ist mittelgross, hager, abgemagert, sieht fast cachektisch aus. An den inneren Organen, speciell den Lungen, ist nichts Abnormes nachweisbar. Das rechte Bein liegt schlaff in Aussenrotation, ist um $4 \mathrm{Cm}$. kürzer als das linke, der rechte Oberschenkel ist fast in ganzer Länge bedeutend dicker, als der linke. Etwa an der Grenze des unteren und mittleren Drittels fühlt man eine starke Verdickung des Knochens. An dieser Stelle ist abnorme Beweglichkeit, aber keine Crepitation nachweisbar. Bei Bewegungen grosse Schmerzhaftigkeit.

1) Neumann, Langenbeck's Archiv. Bd. XXIII. 
Es wird nach diesem Befunde eine Pseudarthrosis femoris infolge Tumorbildung angenommen und zunächst am 13. August 1897 eine Probeincision vorgenommen. Nach Incision der Musculatur quellen weiche, grauröthliche 'Tumor(Sarcom-)massen hervor. Daher schloss Herr Geheimrath $\mathrm{Helferich}$ sofort die Exarticulatio femoris an.

Die Heilung erfolgte in normaler Weise, Pat. geht jetzt mit Prothese umher.

$$
\text { Präparat. }
$$

Nachdem der Femur unter Schonung des Tumors möglichst von Weichtheilen frei präparirt war, ergab sich, dass der Knochen etwas näher dem unteren Ende tracturirt und die Knochenenden durch eine fast kugelrunde Aftermasse verbunden waren. Der Durchmeser des Tumors ist überall etwa $7 \mathrm{Cm}$. (gemessen am gehärteten Präparat).

Die Consistenz des Tumors war sehr weich, die Farbe röthlich-grau, ähnlich, wie eine parenchymatöse Struma, doch nicht so ähnlich, dass dies unbedingt auffallen musste. Colloide Massen waren makroskopisch absolut nicht zu sehen. Ein stark faseriger, zum Theil wie geschichteter Bau fällt besonders an dem nach Kayserling conservirten Präparat auf. Der grösste Theil der Circumferenz wird begrenzt durch eine fast $1 \mathrm{Mm}$. dicke, bindegewebige Kapsel, von der sich die eigentlichen Tumormassen abziehen lassen, und von der direct Muskelfasern abgehen. An der Innenseite findet sich ein auf dem Durchschnitt bis zu $5 \mathrm{Mm}$. breiter Knochen als Begrenzung des Tumors, in der Breite von etwa $3,5 \mathrm{Cm}$., der sich brückenförmig von dem oberen Femurfragment zum unteren um den Tumor herumlagert, aber mit diesen Fragmenten nur mittelst Periostlagen zusammenhängt.

Die Femuriragmente liegen mit ihren Enden in den Tumormassen, d. h. der 'Tumor umgreift oben und unten noch den Femurschaft. Die Fragmente sind ad longitudinem und ad latus dislocirt, insofern das obere mehr urach aussen steht, als das untere. Die Längsdurchschnitte des Femurs zeigen sonst normales Fettmark.

Ein Theil des Tumors wurde sogleich in 4 proc. Formol fixirt, ein Theil erst nach einigen Tagen, nachdem das Präparat erst zwecks Photographiren im Wasser gelegen und darauf nach dem Kayserling'schen Verfahren fixirt war, in Alkohol nachgehärtet.

\section{Mikroskopische Lntersuchuug.}

Die mikroskopischen Bilder aus verschiedenen Theilen des Tumors sind sich nicht immer gleich. Am typischsten scheinen die Theile zu sein, wo sich ein Conglomerat von Hohlraumen von grösserem und kleinerem Durchmesser, im Allgemeinen rundlicher Gestalt, dem Auge präsentirt. Die Begrenzung der Hohlräume geschieht durch eine Reihe gleichmässig tingirter Zellen, deren Zellgrenzen im Allgemeinen sehr undeutlich, zuweilen durch einen helien Spalt angedeutet sind (vergl. Gutknecht, Virch. Archiv. Bd. IC. S. 316). Die Zellen sind meist bedeutend höher, wie breit, oft auch fast cubisch, zuweilen aber auch ganz schmal und lang. Das Protoplasma ist gleichmässig, sehr gut von den Farbstofien tingirt. All Kernen kann man 2 Arten unterscheiden, ohne dass jedoch 
Uebergänge fehlten, oder die beiden Typen stets scharf von einander getrennt werden könnten: erstens dunkel tingirte Kerne, ohne Kernstructur, kleiner als die anderen, meist rond, selten länglich, und zweitens grössere, mehr oder weniger bläschenförmige Kerne, in denen ein zartes Chromatingerüst und zuweilen Kernkörperchen sichtbar sind. Letztere finden sich besonders deutlich in grösseren, zum Theil mehr länglichen Kernen. Die Grösse dieser Kerne variirt mehr, als die der dunkel tingirten. Die Stellung der Kerne ist einmal mehr basal, einmal näher dem Lumen der Hohlräume, oft auch ungefähr in der Mitte der Zellen.

Auf die Reihe einzelner Zellen von dem eben beschriebenen Cha. rakter folgt an typischen Stellen eine Lage platter, länglicher, dunkel tingirter Kerne, deren Längsrichtung aber tangential zum Lumen der IIohlräume liegt, and die sich deutlich als Kerne eines Endothels markiren, das feinste Capillaren von den Epithelringen trennt. Diese Capillaren, die nun zwei derartige Hohlringe von einander trennen, sind oft anf dreieckige Hohlräume reducirt, oder der Capillarraum ist ganz verschwunden, und die 2 Epithelringe sind nur durch eine scharfe Linie von einander getrennt, die sich von Zeit zu Zeit zu einem Endothelkern verdickt. Zuweilen fehlt selbst diese scharfe Linie $\mathrm{zwischen}$ den $\mathrm{zwei}$, einen Hohlraum begrenzenden Epithelschichten, oder nur an einzelnen Stellen ist eine Lücke zwischen ibnen.

An vielen Stellen sind die Hohlränme selbst auf einzelne Lutcken zwischen den Epithelringen reducirt, d. h. wir haben eine doppelte Lage von Epithelzellen, einen Epithelbalken vor uns, der an beiden Seiten durch Endothel-bekleidete Capillaren begrenzt ist. Fehlen letztere wieder, oder sind sie mangelhaft entwickelt, so können noch mohrere Lagen von Epithelzellen ohne begrenzende, resp. trennende Endothelien an einander gelagert werden und, da,' wie gesagt, meist die Zellgrenzen undeutlich oder gar nicht sichtbar sind, einen grossen, vielkernigen Protoplasmaklumpen bilden. Derartige Zellconglomerate bilden an verschiedenen Stellen den wesentlichen Bestandtheil des Tumors.

An anderen Stellen zeigen solche mehrreihige Zellbalken ausgesprochen längliche Form. Sie verleihen dem Tumor auch mikroskopisch eine geschichtete Structur.

An wieder anderen Stellen uberwiegt eine Hoblraumbildung durch Epithelringe, aber ohne dass die Hohlräume irgend nennenswerthe Grösse erreichen.

Endlich giebt es Zellbalken, deren Zellen von Zeit zu Zeit zur Bildung eines kleinen Hohlraumes auseinanderweichen, so dass das langgestreckte Zellconglomerat rosenkranzähnliche Contouren erhält.

In den endothelbegrenzten Capillaren findet sich zuweilen Blut, öfter in ganzer Länge der Kanäle. Einige grössere Gefässe sind im Tumor vorhanden, aber auch meist nur durch eine einfache Endothellage von den Epithelringen getrennt. An einigen Stellen bietet die Abwechselung der gut injicirten Gefässe und der epithelumsäumten Hohlräume ein sehr instructives Bild. Grössere Bindegewebsbalken rufen nur in einigen Schnitten eine Läppchenbildung hervor, sonst scheint das Bindegewebe wesentlich auf die Endothelien reducirt zu sein.

Es ist zu betonen, dass dort, wo die Hohlräume regelmässig und be- 
sonders grösser gebildet sind, in den umgrenzenden Epithelringen die dunkel tingirten kleinen, runden Kerne bei Weitem ulberwiegen. An einigen wenigen Stellen haben dieselben eine ausgesprochen cylindrische Form. Dort wo der Bau atypischer wird, wo Zellenconglomerate und -balken auftreten, überwiegen die hellen, bläschenförmigen Kerne.

In den Hohlräumen fiuden sich nun sehr oft, iu den grösseren fast regelmåssig eigenartige Schollen, die ähnliche Eigenschaften haben, wie das Colloid der Schilddrüse. Man kann alle drei von Gutknecht aufgestellten Colloidarten auffinden, indem sich einzelne Schollen gar nicht färben, die meisten jedoch bei Färbung mit Alauncarmin blass röthlich, doch nicht so stark als das Protoplasma gefärbt erscheinen. Mit Alauncarmin und Leberfärbung mittelst der W eigert'schen Fibrinfärbung erhält man einen blassbläulich-rothen oder dunkelblauen, mit Hämatoxylin oder Hämalaun einen bläulichen, mit Eosin einen röthlichen, jedoch nicht so hell leuchtenden Farbenton, wie das Colloid einer Struma colloides giebt, bei Färbung nach von Gieson einen grünlich-gelben Ton, genau wie das Colloid einer Struma. Bei Färbung mit Hämatoxylin-Eosin sind einige Schollen bläulich, andere roth gefärbt. Endlich finden sich Schollen von runder Form, die eine mehr oder weniger gelbe, resp. gelbbraune Farbe angenommen laben.

Die Gestalt der Schollen ist meist abhängig von der Form der sie einschliessenden Hohlräume. Doch finden sich meist Spalten zwischen dem Epithelkranz und den Schollen, oft auch eine zackige Begrenzung der Schollen oder endlich eine kreisrunde Form derselben, unabhängig von der Form des einschliessenden Epithelringes. Zuweilen zeigen die Hohlräume ein Netzwerk, das im Centrum eine derartige, mehr oder weniger runde Scholle beherbergt. Letztere ist dann öfter gelb gefürbt, $d$. h. hat wohl seine Eigenfarbe bewahrt, während die dem Epithelring näher liegenden Theile des Hohlraumes von einer anscheinend zum Theil ausgelaugten, d. h. Lücken enthaltenden Masse blau (Hämatoxylin) oder roth (Alauncarmin) tingirten Colloids eingenommen sind. ${ }^{1}$ )

\section{Classification.}

Nach dem histologischen Verhalten des Tumors müssen wir $e_{B}$ als das wahrscheinlicbste ansehen, dass wir die Metastase einer Schilddruise vor uns haben, zumal Pat. eine mässig grosse, beiderseits entwickelte Struma von ziemlich fester Consistenz hat, die sich be ihr vor etwa 30 Jahren ziemlich schnell entwickelt hat, aber seitdem auf der augenblicklichen Grösse steben geblieben ist. Die nähere Classification der Geschwulst ergiebt sich ohne Weiteres aus ihrem Bau.

Für die Diagnose Carcinom künnte einerseits eine gewisse Polymorphie der die soliden Zellbalken zusammensetzenden Epithelien sprechen, doch ist diese Polymorphie (mosaikartiges Ausseben der

1) Aehnliches erwăhnt Wölfler, Langenbeck's Archiv. Bd. XXIX. S. 55. 
Zellstränge) auch von der sicher gutartigen Struma beschrieben. Andererseits finden sich an einzelnen Stellen des Tumors Bilder, die ohne Zweifel eine alveoläre, d. b. carcinomähnliche Structur haben. Es finden sich hier etwas grössere Bindegewebsbalken, die das Geschwulstgewebe, das die obeu beschriebenen histologischen Charaktere aufweist, in grössere Läppchen theilen, und in diesen Zellbalken einzelne solide Epithelnester neben einander, äbnlich wie es $\mathrm{Hä} \mathrm{ck}$ el in der seiner Mittheilung beigegebenen Figur wiedergiebt. Soll man wegen dieser ganz vereinzelten Stellen den ganzen Tumor als Carcinom bezeichnen? Bei der verschwindend geringen Menge derartiger Bilder, bei der Tendenz auch dieser soliden Epithelnester, sich zu Hoblräumen umzumodeln, so dass wir also die alveoläre Structur nur für eine vorubergehende Uebergangsbildung halten können, bei dem erdrickend uberwiegenden adenomartigen Bau des Tumors, bei dem Feblen jeglicher kleinzelliger Infiltration, bei der klinischen, relativen Gutartigkeit des Tumors scheint die Rangirung unter die Adenomel) berechtigter, unter die Adenome, die v. Eiselsberg speciell beim Vorkommen von Metastasen als Adenocarcinome bezeichnen will (ct. Discussion auf dem Chirurgencongress 1893).

Für eine Metastase eines primären Schilddrüsentamors spricht in unserem Fall neben der Structur das langsame Wachsthum des Tumors, der doch wabrscheinlich schon vor 21/2 Jahren vorhanden war. Es ist wobl sicher, dass der Tumor die Ursache des Nichtheilens der Fractur nach dem 2. Fall war. Ob der Tumor schon den 1. Bruch veranlasste, ist nicht ohne Weiteres sicher. Dagegen könnte die Consolidation der Fractur nach dem 1. Unfall sprechen. Es wïrde sich dann ein im Blut kreisender Keim der Scbilddrtise nach der ersten Fractur in derselben als einem Locus minoris resistentiae festgesetzt und hier bei der Schwellung und Hyperämie bei der Callusbildung die geeignete Nahrung zum Weiterwachsen gefunden haben. Es ist leicht verständlich, dass trotzdem die Fractur zuerst zur Consolidation kam, da Callus und Tumor sicb zu gleicher Zeit entwickelten, und ersterer wohl nicht sogleich dem Tumor zum Opfer fiel. Nachträglich aber genügte der, immerhin stärkeren Insulten nicht gewachsene, Callus nicht der Gewalt bei einem Fall auf das Strassenpflaster. Mit dieser Deutung würde die Behauptung stimmen, dass Strumametastasen sich öfter in Fracturen festsetzen (ef. z. B. den Fall I von Cramer, Langenbeck's Archiv Bd. XXXVI). Aber sollte nicht umgekehrt oft die Strumametastase die Ursache der Fractur sein?

1) Wölfler, Langenbeck's Archiv. Bd.XXIX. S. 60. 
In unserem Falle könnte sebr wohl ein kleiner Tumor bei der ersten Fractur schon vorbanden gewesen sein und durch Sehwächung des Knochens das Zustandekommen der Fractur erleichtert, die Callusbildung aber zunächst noch nicht so gehindert haben, um eine wenn auch wenig widerstandsfähige Consolidation zu ermöglichen. Der von Middeldorpf (Langenbeck's Archiv Bd. XLVIII) publicirte Fall spricht für diese Ansicht.

Jedenfalls können wir also den Beginn der Erkrankung schon 2 Jahre zurickschreiben. Dieses langsame Wachsthum stimmt mit den Beobachtungen bei anderen Strumametastasen uberein. Ich verweise nur auf die beigelegte Tabelle. Andere maligne Tumoren, speciell Carcinome, wachsen meist rascher. Bei dem ausserordentlichen Zellreichthum unseres Tumors, der ja nur minimale Massen Bindegewebe beberbergt, besteht eben eine grosse Incongruenz zwischen klinischem Verlauf und histologischen Bild, eine Incongruenz, die fast nur die Tumoren der Schilddrüse aufweisen.

Was auch fiir eine Strumametastase zu sprechen scheint, ist ein zutälliger, aber um so interessanterer Befund im makroskopisch ganz normalen Fettmark des Femur etwa in der Mitte zwischen der Geschwulst und der unteren Gelenkfläche des Femur. Hier fand sich nämlich eine fast mikrosls op ische, we i tere Strumametas tase. Vergleicbshalber batte ich ein Stiick Fettmark aus diesem Theil des Femur entkalkt und geschnitten. Schon an dem eingebetteten Stuckchen fiel eine consistentere Masse vou ca. 1:3 Mm. Querscbnitt inmitten des porösen Fettmarkes auf. Und in Wirklichkeit zeigte das Mikroskop zwischen normalem Fettmark, aber scharf von Letzterem getrennt, eine compactere Masse, die leider meistens sehr schlecht conservirt war. Sie bestand vorzugsweise nur aus mit den Farbstoffen diffus gefärbten Schollen, zwischen denen gelbliche Massen (ausgelaugtes Blut) sichtbar waren. Inmitten der compacteren Massen fielen schon makroskopisch drei kreisrunde kleine Oeffnungen auf, die von einem amorphen, vom Farbstoff diffus tingirten Ring umgeben waren. An einzelnen, wenigen stellen sieht man nun aber die wirkliche Structur erhalten, nähmlich schöne, genau den Tumorzellenbalken gleichende, gut tingirte Zellreihen mit runden, dunklen Kernen. Einmal ist auch ein von einem gut conservirten Epithelring umsäumtes, kreisrundes Lumeu nachweisbar.

Es ist der Befund dieser weiteren wohl mikroskopisch zu nennenden Metastase nicht uninterressant bei der Frage des therapeutischen Vorgebens bei den malignen Tumoren der langen Röhrenknochen. Allerdings wird es sich meistens dabei um primäre Tumoren bandeln. 
Bekanntlich sind in neuerer Zeit Versuche mit Resectionen der Knochen in der Continuität auch bei Tumoren gemacht, die fruber die Amputation, resp. Exarticulation indicirten. Herr Geheimrath Helferich ist im Allgemeinen kein Freund dieser Resectionen, wenn er auch, notbgedrungen, z. B. bei mebreren Tibiasarcomen am unteren Ende die Resection mit nachfolgender Implantation der Tibia oder der Fibula in den Talus gemacht bat. Fälle wie der meinige oder ein anderer hier beobachteter, bei dem ein Tibiasarcom zu einer Metastase in der unteren Femurepiphyse gefibhrt hatte, mahnen immerhin zu grosser Vorsicht bei den oben erwähnten therapeutischen Eingriffen.

Der Knochen, welcher einen Theil des Ueberzuges des Tumors bildet, bietet die Erscheinungen dar, wie wir sie oft in der Nähe von destruirenden Processen im Knochensystem finden: starke Neubildung, Erweiterung der H a vers'schen Kanälchen durch Bindegewebe und Bildung eines Kranzes von Osteoblasten. Der Tumor ist vom Knochen noch durch eine riemlich breite Bindegewebslage getrennt. Einige Howship'sche Lakunen mit Osteoblasten deuten auf resorbirende Processe hin. Die Knochenbälkchen umschliessen an einigen Stellen Knorpelinseln.

Pathologisch-anatomisch durften folgende Beobachtungen von Interesse sein. Zunächst das Vorkommen von Colloid in der Metastase. M. B. Sch midt hat bekanntlich vor kurzem den ,Secretionsvorgängen in Krebsen der Schilddrüse und der Leber und ihren Metastasen" einen Aufsatz in Virchow's Archiv Bd. CXLVIII gewidmet, und v. Eiselsberg') konnte in einer bemerkenswerthen Mittheilung es wahrscheinlich machen, dass eine Strunametastase im Sternum vicariirend für die extirpirte strumöse Schilddrüse eintrat. Unser Tumor zeigt eine Erscheinung, die, so viel ich weiss, noch nicht in Strumametastasen beobachtet ist. Man sieht nämlich an verschiedenen Stellen auch die interfolliculären Capillaren mit einer Masse geftullt, die ähnliche Reactionen, wie das Colloid innerhalb der Follikel giebt. Mehrmals erscheinen die Endothelbekleideten Hohlräume durch diese Massen sehr ausgedehnt, und inmitten der Massen in einem Hohlraum finden sich rothe Blutkörperchen. Letateres Verbalten scheint mir allerdings mehr fur ein Kunstprodukt zu sprechen, da man sonst wobl eine innigere Vermischung der Massen und der rothen Blutkörperchen erwarten könnte. Andere Stellen aber lassen bei dem absolut klaren mikroskopischen Bild und bei der Vorsicht, die bei der Fixation gebraucht ist, doch auf die

1) v. Eiselsberg, Langenbeck's Archiv. Bd. XLVlII. 
Möglichkeit eines Uebertrittes von colloidähnlichen Massen in die Capillaren (Lymph, Blutcapillaren?) schliessen.1)

An einzelnen Stellen ist auch inmitten grösserer Gefässe neben Blut eine an Colloid erinnernde, ganz fein gekörnte oder homogene Masse vorhanden. Dass diese Bilder nicht etwa eine hyaline Metamorphose des Bindegewebes zur Grundlage baben können, möchte ich noch besonders betonen.

Diese Structurbilder erinnern an Beobachtungen Langhans") (und seiner Schule: Gutknecht ${ }^{3}$ ), Marie Zielinska ${ }^{4}$ ), woselbst auch die übrigen Literaturangaben hiertiber nachzusehen sind). L a nghans betont das Vorkommen von Gefässlumina, die ganz mit blassem Colloid gefullt sind. „Die normale Thyreoidea scheint sich in dieser Beziehung ganz cbenso zu verhalten, wie die Struma, während sonst die Adenome als nicht functionirend angesehen werden können."

Der andere Punkt von Interesse ist das Verbalten der beiden beschriebenen Kernarten. Während die kleinen, dunkel tingirten, im Ganzen runden Kerne gerade in den Theilen überwiegen, die den normalen Bau der Schilddruse am ausgesprocbendsten nachahmen, fanden sich die hellen, bläschenförmigen grossen Kerne wesentlich und überwiegend in den atypischer gebauten Theilen, dort wo keine Follikel gebildet sind, sondern meist nur Zellbalken und \%ellconglo. merate die Bestandtheile des Tumors bilden. Kommt in diesen Theilen einmal ein follikelähnliches Gebilde zum Vorschein, so uberwiegen in dem begrenzenden Epithelsaum gleich wiederum die dunkeltingirten Kerne.

Bei einer Durchsicht der Literatur fällt mir eine Beschreibung auf, welche ein Analogon zu dem von uns beobachteten Kernverhalten darbietet. Ribberts) sagt bei der Beschreibung eines regenerirten, künstlichen Defects der Hundethyreoidea: „Ueberall kann man die neugebildeten Alveolen leicht von dem alten Drüsengewebe unterscheiden. In diesem sind die Epithelien niedrig, ibre Kerne intensiv und gleichmässig gefärbt und klein, doch (soll offenbar heissen: dort) haben die Epitbelien fast die doppelte Höhe, und auch ibre Kerne sind grösser, blasser gefärbt, bläschenförmig."

An drei untersuchten Strumen fand ich keinen solchen auffallen-

1) Vgl. auch die Beobachtung von M. B. Schmidt, Virchow's Archiv. Bd. CXIAVIII. S. 59 .

2) Laughans, Virchow's Arcbiv. Bd. CXXVIII. S. 389.

3) Gutknecht, Virchow's Archiv. Bd. IC. S. 423.

4) Marie Zielinska, Virchow's Archiv. Bd. CXXXVI. S. 172ff.

5) Ribbert, Virchow's Archiv. Bd. CXVII. 
den Unterschied zwischen den Kernen. Die Schilderung, welche Wölfler (Ueber die Entwicklang und den Bau der Schilddrüse, Berlin 1880) von bestimmten Arten von Drüsenbläschen der werdenden Schilddrtse giebt, erinnert auch an die beschriebenen Verhältnisse, ebenso wie seine Fig. 37, Tafel VII (ebendaselbst) an das Bild vieler Partien unseres Tumors, speciell, was auch die meist mebr cylindrische, als cubische Gestaltung der Zellen betrifft.

Aehnliche, publicirte Fälle.

Die beigegebenen Tabellen (s. S. 358 u.f.) geben eine Uebersicht Uber die bisher, soweit mir bekannt, publicirten, operativ behandelten Struma . metastasen im Knochen. Es sind nur die Fälle genommen, die unserem Tumor ähnlich scheinen, d. h. mikroskopisch neben der Structur der Schilddrüse einen adenomatösen, keinen ausgesprochen carcinomatösen Bau oder gar sarcomatösen Bau aufwiesen. Es sind in früherer und Ietzterer Zeit verschiedentlich Fälle der letzteren Art als „Strumametastasen" publicirt. Dass Carcinome oder Sarcome der Glandula thyreoidea metastasiren, dass sie auch (cf. z. B. Ka u fm an n, Deutsche Zeitschr. f. Chir. Bd. XI und XIV) mit Vorliebe nächst den Lungen in den Knochen metastasiren, ist nach dem Analogon bei den tibrigen Organen nicht wunderbar. Das, was der Schilddrise eigen und bisher auch nicht aufgeklärt ist, ist eben die Incongruenz zwischen klinischem Verhalten und histologischem Bau, wie es besonders Wölfler, v. Eiselsberg, M. B. Schmidt u. s. w. betonen.

Von diesen metastatischen Adenomen, Wölfler's Adenocarcinomen der Glandula thyreoidea konnte ich, wie die beifolgende Tabelle lehrt, fünfzehn operativ behandelte Fälle in der Literatur finden. Der Fall Gudenius von Cramer, (Langenbeck's Archiv Bd. XXXVI) gehört nicht hierher. Die grosse Strumametastase im Sternum wurde ihrer Pulsation balber für ein Aneurysma gehalten und der immensen Schmerzen halber, die die Pat. erlitt, mit successiver Unterbindung der grossen Gefässe behandelt.

Die Tabelle lehrt das liberwiegende Vorkowmen bei Frauen (13:2 Männern). Die Zahlen entsprechen jedoch sicher nicht den thatsächlichen Verhältnissen; v. Eiselsberg1) z. B. publicirte eine Anzahl, per autbopsiam bewahrheiteter Fälle, die fast nur (6 von 7) Männer betrafen.2)

Die Patienten befanden sich meist in höherem Alter.

Sehr oft waren die Metastasen am Scbädel localisirt.

1) v. Eisels berg, Langenbeck's Archiv. Bd. XLVI.

2) Vgl. die 'Tabelle Jäger's, Bruns' Beiträge. Bd. XIX. Heft 3. 
Die Tumoren waren meist langsam gewachsen und zeigten auch in den Recidiven nach der Operation relative Gutartigkeit.

Eine sichtbare Struma bestand meist neben dem Tumor (in zehn Fällen), nur ein Fall hatte weder einen sichtbaren, noch fühlbaren Kropf. Die Struma war überwiegend von Jugend auf vorhanden gewesen oder machte sich vor der Metastase bemerklich. Es ist zu betonen, dass mehrere (bei Sectionen beobachtete) Fälle von Adenommetastasen der Glandula thyreoidea publicirt sind, bei denen in vivo keine Struma und auch in mortuo keine vergrösserte Schilddrüse gefunden wurde, wohl aber in Letzterer kleine (erbsengrosse) Adenomknötchen.

Die Symptome waren die eines gewöhnlichen Knochentumors. Doch muss die Schmerzhaftigkeit und das Symptom der Pulsation, auf das besonders Cramer und Feurer aufmerksam machten, endlich das langsame Wachsthum an eine Strumametastase, speciell bei bestehendem Blähhals, denken lassen, bei der Operation selbst die meist abundante Blutung. Ein Symptom, das v. Eiselsberg') bei der Metastase eines als Cylinderzellencarcinom metastasirten Adenoms der Schilddrüse beobachtete, eine Anschwellung des Tumors während der Menses, ist von keinem anderen Autor erwähnt.

Die Prognose der Operation bei solitären Metastasen, und nur solche kommen wohl zur Operation, ist eine gute. Die unmittelbaren Operationsresultate sind bei den Fällen, wo man entternt vom Tumor arbeitet (bei Amputationen, Exarticulationen) absolut gut, dort wo der Tumor selbst im Operationsgebiet liegt, wegen der starken Blutung weniger gut (cf. Fall 2 der Tabelle). Auch die Recidive bleiben meist aus oder kommen sehr spät, dann allerdings meist multipel.

Zu warnen ist nach unseren mikroskopischen Untersuchungen wobl vor einer Continuitätsresection langer Röhrenknochen.

Eine nicht ohne Weiteres zu beantwortende Frage ist die nach der Extirpation der Schilddrïse, wenn sich die Metastase als eine solehe dieser Drüse erwiesen hat. Die Erfahrungen der Operateure, welche die Struma ruhig sitzen liessen, so Kraske (Fall 7), Riedel (Fall 9), sprechen nicht gegen dieses Verfahren. Umgekehrt spricht eine Erfahrung E wald's (Fall 5) und Cramer's ${ }^{2}$ ) gegen die sofortige Strumectomie. Dieselben denken nämlich daran, dass gerade die Extirpation der Struma Anlass zur Metastasirung gegeben haben kann. Allerdings ist dies nur eine Vermuthung.

1) Eiselsberg, Langenbeck's Archiv. Bd. XLVIII.

2) Cramer, Langenbeck's Archiv. Bd. XXXVL. 
I. Struma metastasen

\begin{tabular}{|c|c|c|c|c|c|c|}
\hline$\underset{g}{\stackrel{g}{g}}$ & Name des Autors & 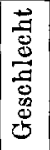 & 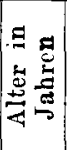 & Metastasen in: & $\begin{array}{l}\text { Seit wann } \\
\text { bemerkt? }\end{array}$ & Symptome der Metastaser \\
\hline 1 & $\begin{array}{c}v . \text { Eiselsberg } \\
\text { (Langenbeek's Archiv. } \\
\text { Bd. XLVI). }\end{array}$ & $\mathrm{m}$ & 38 & Schädel. & 4 Jahr. & $\begin{array}{l}\text { Beim Bucken grösse: } \\
\text { werdend, keine Meta } \\
\text { stasen. }\end{array}$ \\
\hline 2 & $\begin{array}{l}\text { v. Eiselsberg } \\
\text { (Ebenda). }\end{array}$ & $\mathbf{m}$. & 32 & Manubrium sterni. & $6 \mathrm{Jahr}$. & $\begin{array}{l}\text { Entstand 3 Monate nacl } \\
\text { einem Stoss auf di } \\
\text { Brust. - Seit einiger } \\
\text { Monaten laneinirend } \\
\text { Schmerzen. }\end{array}$ \\
\hline $\mathbf{3}$ & $\begin{array}{l}\text { M. B. Schmidt } \\
\text { (Virchow's Archiv } \\
\text { Bd. CXLVIII). }\end{array}$ & w. & $6 \mathrm{~L}$ & $\begin{array}{l}\text { Stirn. Pleura. } \\
\text { Femur. }\end{array}$ & 6 Mon. & - \\
\hline 4 & $\begin{array}{c}\text { v. Hofmann } \\
\text { (Wien. klin. Wochenschr. } \\
\text { 1897. Nr. 46), } \\
\text { Ewald } \\
\text { (Wien. klin. Wochenschr } \\
\text { 1893. S. 458). }\end{array}$ & w. & 26 & L. Jochbein. & $13 \mathrm{Jahr}$. & $\begin{array}{l}\text { Angeblich nach einem } \\
\text { Faustschlag ins Ge- } \\
\text { sicht, 14 Tage nach } \\
\text { dem Insult. }\end{array}$ \\
\hline $\mathbf{5}$ & $\begin{array}{l}\text { v. Hofmann (Evoald) } \\
\text { (Ebenda). }\end{array}$ & w. & 43 & Scapula. & $\begin{array}{l}\text { Bald nach } \\
\text { der Ope- } \\
\text { ration der } \\
\text { Struma. }\end{array}$ & $\begin{array}{l}\text { Schmerzen im rechten } \\
\text { Arm, besonders bej } \\
\text { Bewegungen. }\end{array}$ \\
\hline 6 & $\begin{array}{c}\text { Wölfler } \\
\text { (Langenbeck's Archiv. } \\
\text { Bd. XXIX). }\end{array}$ & w. & 57 & Stira. & I Jahr. & Kopfschmerzen. \\
\hline 7 & $\begin{array}{c}\text { Kraske } \\
\text { (Chir. Congress 1893) u. } \\
\text { Boris-Bontsch-Ormo- } \\
\text { lowsky } \\
\text { (Dissert. Freiburg 1893). }\end{array}$ & w. & 53 & Stirnbein. & $\begin{array}{c}\text { 4-6 } \\
\text { Wochen. }\end{array}$ & $\begin{array}{l}\text { War rasch entstanden, } \\
\text { wie Krask e betont, u. } \\
\text { zwar nach Bontsch } \\
\text { an einer Stelle, die } \\
\text { vor } 1 / 2 \text { Jahr ein Trau- } \\
\text { ma erlitten hatte. }\end{array}$ \\
\hline 8 & $\begin{array}{c}\text { Riedel } \\
\text { (Deutsche Zeitsohr. f. Chir. } \\
\text { Bd. XV u. Chir. Congr. } \\
\text { 1893). }\end{array}$ & w. & 40 & $\begin{array}{l}\text { Unterkiefer, u. zwar } \\
\text { an der Aussenseite } \\
\text { des Kiefers. }\end{array}$ & $\begin{array}{c}\text { Seit } 7 \\
\text { Jahren. } \\
\text { Vor 3 } \\
\text { Jahren un- } \\
\text { vollständig } \\
\text { operirt. }\end{array}$ & - \\
\hline
\end{tabular}


die radical entfernt wurden.

\begin{tabular}{|c|c|c|c|c|c|}
\hline $\begin{array}{l}\text { Mikroskopische } \\
\text { Structur der } \\
\text { Metastasen }\end{array}$ & $\begin{array}{l}\text { Struma vor- } \\
\text { handen? }\end{array}$ & Seit wann? & $\begin{array}{l}\text { Mikroskopische } \\
\text { Structur der } \\
\text { Struma }\end{array}$ & Verlauf & Bemerkungen \\
\hline Adenom. & Markiger Kropf. & Seit 20 Jahren. & $?$ & $\begin{array}{l}\text { Nach } 4 \text { Jahren } \\
\text { locales Recidiv. } \\
\text { Lebte noch nach } \\
8 \text { Jahren. }\end{array}$ & - \\
\hline $\begin{array}{c}\text { Adenocarcinom, } \\
\text { aber nirgends } \\
\text { wirkliche carci- } \\
\text { nomatöse } \\
\text { Structur. }\end{array}$ & Kropf. & $\begin{array}{l}\text { Seit der } \\
\text { Jugend. }\end{array}$ & $\begin{array}{l}\text { In der Schild- } \\
\text { druse multiple } \\
\text { Adenome. }\end{array}$ & $\begin{array}{c}+1 / 4 \text { Stunde p. } \\
\text { operationem an } \\
\text { Anämie. }\end{array}$ & $\begin{array}{l}\text { Continuitätsinfec- } \\
\text { tion (Kauf - } \\
\text { mann)? }\end{array}$ \\
\hline $\begin{array}{l}\text { Adenom, resp. } \\
\text { Adenocarcinom. }\end{array}$ & Struma rechts. & $?$ & $\begin{array}{c}\text { Knoten von } \\
\text { folliculärem } \\
\text { Schilddrusen- } \\
\text { bau, solche aus } \\
\text { Cylinderzellen- } \\
\text { schläuchen und } \\
\text { solche mit soli- } \\
\text { den epitbelialen } \\
\text { Strängen. }\end{array}$ & $\begin{array}{l}\text { † } 4 \text { Tage nach } \\
\text { Opcration der } \\
\text { Sohädelmeta- } \\
\text { stase im Coma. }\end{array}$ & - \\
\hline $\begin{array}{c}\text { Metastase } \\
\text { des Adenocar- } \\
\text { cinoms. }\end{array}$ & $\begin{array}{c}\text { Struma rechts } \\
\text { (später } \\
\text { enuoleirt). }\end{array}$ & $\begin{array}{c}\text { Seit } 11 \text { Jahren } \\
\text { Dickerwerden } \\
\text { des Halses, } \\
\text { seit } 5 \text { Jahren } \\
\text { Geschwulst } \\
\text { rechts be- } \\
\text { merkt. }\end{array}$ & Adenocarcinom. & $?$ & - \\
\hline Adenocarcinom. & $\begin{array}{l}\text { Rechts orange- } \\
\text { grosser Tumor } \\
\text { (enucleirt } \\
\text { zuerst). }\end{array}$ & 4 Jahr. & Gallertkropf. & $\begin{array}{c}+4^{1 / 2} \mathrm{Jabr} \text { post } \\
\text { strumectomiam. } \\
\text { Vielleicht } \\
\text { locales Struma- } \\
\text { recidiv! }\end{array}$ & $\begin{array}{l}\text { Der Fall könnte } \\
\text { fur Metastasirung } \\
\text { nach Eingriffen } \\
\text { sprechen } \\
\text { (cf. Cramer, } \\
\text { Langenb. Arch. } \\
\text { Bd. XXXVI). }\end{array}$ \\
\hline $\begin{array}{l}\text { Struetur des } \\
\text { interacinösen } \\
\text { Adenoms der } \\
\text { Schilddruse. }\end{array}$ & $\begin{array}{l}\text { linke Schild- } \\
\text { drusenhälfte } \\
\text { kleinfaustgross, } \\
\text { knotig, hart, } \\
\text { wenig beweg- } \\
\text { lich. }\end{array}$ & $\begin{array}{c}\text { Vor dem } \\
\text { Stirnknoten. }\end{array}$ & Nicbt exstirpirt. & $\begin{array}{c}f \text { ca. } 4-6 . \text { Mon. } \\
\text { p. oper. }\end{array}$ & - \\
\hline $\begin{array}{c}\text { Adenom } \\
\text { (Struma hyper- } \\
\text { plastica). }\end{array}$ & Struma. & $\begin{array}{c}\text { Bestand schon } \\
\text { bei der Ope- } \\
\text { ration. }\end{array}$ & dito. & $\begin{array}{c}\text { Lebte } 1893 \\
\text { (nach } 3 \text { Jahren) } \\
\text { noch ohne Re- } \\
\text { cidiv, Struma } \\
\text { nicht verändert. }\end{array}$ & $\rightarrow$ \\
\hline $\begin{array}{l}\text { Schilddrusen- } \\
\text { gewebe? }\end{array}$ & $\begin{array}{c}\text { Keine Struma, } \\
\text { auch nicht beim } \\
\text { Auftreten des } \\
\text { Recidiva. }\end{array}$ & - & - & $\begin{array}{l}\text { Nach } 10 \text { Jahren } \\
\text { wieder An- } \\
\text { schwellung des } \\
\text { Kiefers. }\end{array}$ & $\begin{array}{c}\text { Zuerst fur ein } \\
\text { Drusencarcinom } \\
\text { gehalten. }\end{array}$ \\
\hline
\end{tabular}




\begin{tabular}{|c|c|c|c|c|c|c|}
\hline 离 & Name des Autors & 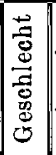 & . & Metastasen in: & $\begin{array}{l}\text { Seit wann } \\
\text { bemerkt? }\end{array}$ & Symptome der Metastase \\
\hline 9 & $\begin{array}{c}\text { Haeckel } \\
\text { 1Correspondenzblatt des } \\
\text { allgem. ärztl. Vereins für } \\
\text { Thüringen 1889) u. } \\
\text { Riedel } \\
\text { (Chir. Congr. 1893). }\end{array}$ & w. & 49 & Unterkiefer. & 1/4 Jahr. & $\begin{array}{l}\text { Vor } 3 \text { Jahren Nerven } \\
\text { schmerzen in der rech } \\
\text { ten Kopfbälfte, vo } \\
1 / 4 \text { Jahre äbnlich } \\
\text { Schmerzen im rechte } \\
\text { Cnterkiefer. }\end{array}$ \\
\hline 10 & Helferich. & w. & 54 & Femur. & $21 / 2 \mathrm{Jahr}$. & $\begin{array}{l}\text { Wiederholte Fractur } \\
\text { Pscudartbrosis. } \\
\text { Schmerzen. Unfähig. } \\
\text { keit zu Gehen. }\end{array}$ \\
\hline 11 & $\begin{array}{c}\text { Hulchinson } \\
\text { (Cylindrical cancer of the } \\
\text { Humerus, Transactions of } \\
\text { the path. Soc. of Liondon. } \\
\text { Bd. XXXVII. 1886). }\end{array}$ & w. & 50 & Humerus. & $\begin{array}{l}\text { Wahr- } \\
\text { scheinlich } \\
\text { bestehend } \\
\text { seit } 5 \text { Mo- } \\
\text { naten. }\end{array}$ & $\begin{array}{l}\text { Schmerzen naci } F a l \\
\text { gegen einen Tiseh. }\end{array}$ \\
\hline
\end{tabular}

II. Strumametastasen,

\begin{tabular}{|c|c|c|c|c|c|c|}
\hline 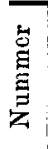 & Name des Autors & 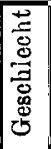 & 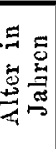 & Ort der Metastase & Dauer & Aetiologie, Symptome \\
\hline 1 & $\begin{array}{c}\text { Cohnheim } \\
\text { (Virchow's Archiv. } \\
\text { Bd. LXVIII). }\end{array}$ & w. & 35 & $\begin{array}{l}\text { Abseedirter Tumor } \\
\text { der Ileosacraljunctur } \\
\text { (Os ilei). }\end{array}$ & $\begin{array}{c}\text { Seit etwa } \\
7 \text { Mon. }\end{array}$ & Dumpfe Schmerzen \\
\hline 2 & $\begin{array}{c}\text { Feurer } \\
\text { (Festschrift f. Ko cher- } \\
\text { Bern. 1891). }\end{array}$ & w. & 53 & Linkes Os parietale. & $\begin{array}{l}3 / 4 \text { bis } \\
1 \mathrm{Jahr} .\end{array}$ & $\begin{array}{l}\text { Schmerzen im linken } \\
\text { Scheitelbein nach Stoss } \\
\text { auf diese Gegend. An- } \\
\text { schwellung nach er- } \\
\text { neutem Trauma } 1 / 4 \mathrm{Jahr} \\
\text { später. }\end{array}$ \\
\hline 3 & $\begin{array}{c}\text { Gussenbauer } \\
\text { (Chir. Congress 1893). }\end{array}$ & w. & $?$ & $\begin{array}{l}\text { 10. ‥ 11. Rucken- } \\
\text { wirbel. }\end{array}$ & $1 / 2 \mathrm{Jahr}$. & $\begin{array}{l}\text { Ischialgische Schmerzen } \\
\text { in beiden Extremi- } \\
\text { täten, später Para- } \\
\text { plegie. }\end{array}$ \\
\hline 4 & $\begin{array}{l}\text { Krönlein } \\
\text { (Jäger in Bruns' Beitr. } \\
\text { Bd. XIX). }\end{array}$ & w. & 69 & $\begin{array}{l}\text { 1. 6. u. } 7 \text {. Halswirbel } \\
\text { u. 1. Brustwirbel. } \\
\text { 2. 3. u. 4. Lenden- } \\
\text { wirbel. }\end{array}$ & $\begin{array}{l}\text { gegend, s } \\
\text { der Dreh }\end{array}$ & $\begin{array}{l}\text { Sturz von einem klei- } \\
\text { nen Wagen mit dem } \\
\text { Rucken auf die harte } \\
\text { Strasse; }{ }^{1}{ }^{\prime} \text { Jahr später } \\
\text { Tumor bemerkt. Seit } \\
4 \text { Monaten Anschwel- } \\
\text { lung in der Nacken- } \\
\text { merzen, Beschränkungen } \\
\text { wegungen des Kopfes. }\end{array}$ \\
\hline
\end{tabular}


Ueber eine Geschwulst von schilddrüsenartigem Bau im Femur.

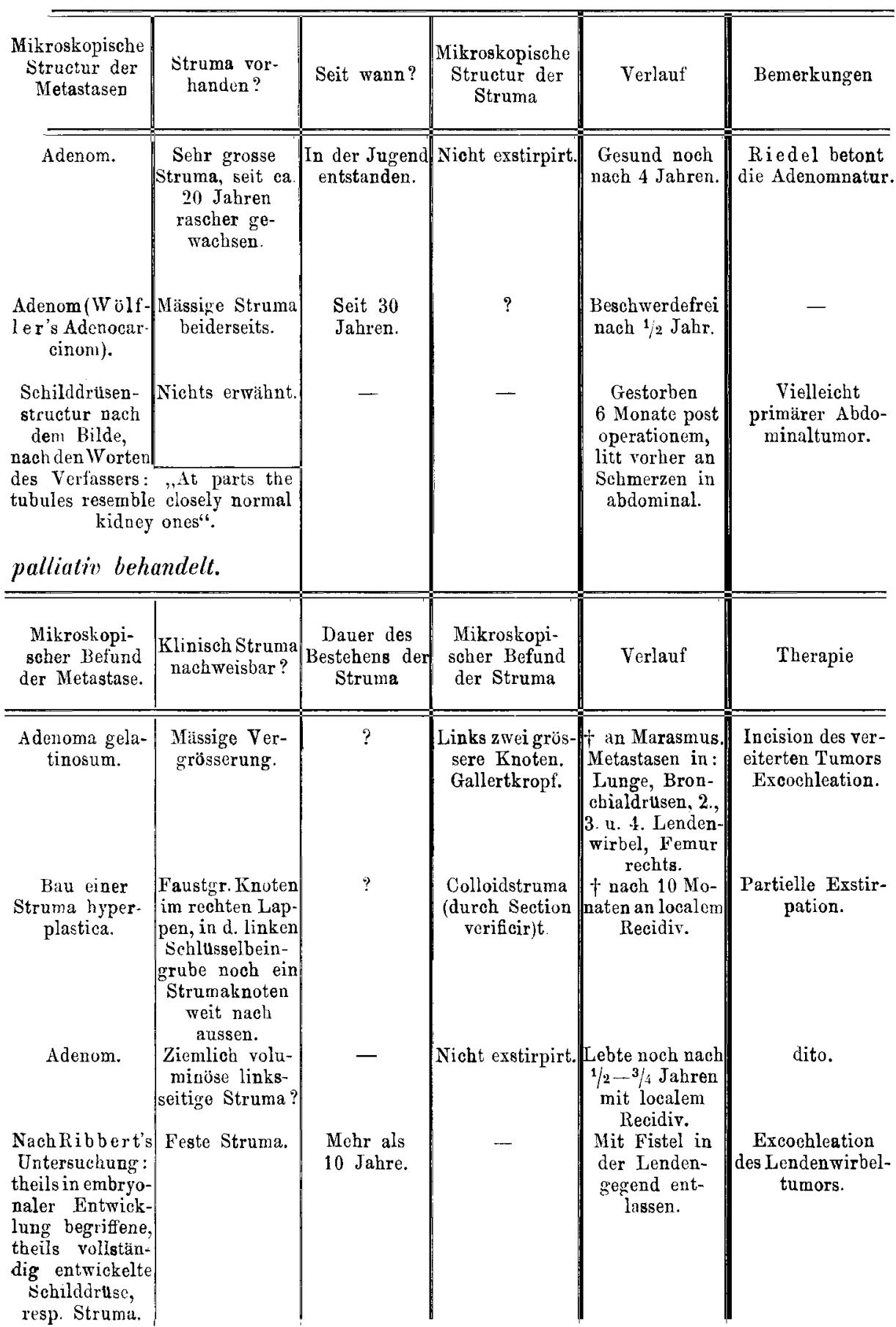


Bezüglich weiterer Details verweise ich auf die Arbeit Jäger's in Bruns' Beiträgen (Bd. XIX), die gerade nach Vollendung dieses Manuscriptes - dasselbe diente als Grundlage für einen am 8 . Januar im Greifswalder medicinischen Verein gehaltenen kurzen Vortrag mit Demonstration des Tumors - erschien. Jäger findet in der Literatur 15 Fälle von Strumametastasen mit krebsigen Partien, 12 Fälle von solchen ohne krebsige Partie, wobei er die nicht operativ behandelten Fälle natürlich mitzählt; dazu kommt noch der Fall von M. B. Schmidt, von Morris (Transactions of the path. soc. of London Vol. XXXI, S. 259) und die Fälle von v. Hofmann (Ewald); auch Hinterstoisser (Festschrift für Billroth 1892) soll Fälle, die hierhin gehören, publicirt haben, doch war mir die Arbeit nicht zugängig. Jäger rechnet auch den Feurer'schen Fall unter die, welche Carcinomgewebe enthielten. Das geht aus der Feurer'schen und Lang b a n s'schen Beschreibung, trotzdem Letzterer den Ausdruck "Metastase eines Carcinoms der Schilddruse" gebraucht, nicht hervor.

Weiterhin betont Jäger die auch uns auffallende Thatsache, dass von den 12 Fällen von Kropfmetastasen ohne carcinomatöse Stellen 9 Fälle auf Frauen und nur drei auf Männer kommen, während bei Kropfmetastasen mit carcinomatösen Stellen 7 Männer und 5 Frauen notirt sind. Wenn Jäger ferner glaubt, dass man wohl mit Fe urer annehmen kann, dass ein Trauma zum ätiologischen Gelegenheitsmoment für Strumametastasen werden kann (6 Fälle von 31 geben ein Trauma an, in 10 Fällen von diesen 31 fehlen anamnestische Daten), so muss man sich dagegen wohl doch noch etwas skeptisch verhalten.

Auf meine Bitte hin sandte mir mein hochverehrter Lehrer und fruherer Chef, Herr Prof. Rib ber t, zwei kleine Stückchen des Jäg er schen Tumors. Dieselben waren in Alkohol fixirt und zeigten makroskopisch im gehärteten Zustand eine grauweissliche Schnittfläche, die makroskopisch einen undeutlich porösen Bau aufwies. Mikroskopisch ergaben sich nicht unwesentliche Unterschiede von meinem Tumor, die allerdings z. Th. auf die verschiedene Fixirung zurückzuführen sein durften.

Zunächst sind die Epithelien durchweg cubisch, ja recht oft bei Begrenzung grösserer Hohlräume abgeplattet. Cylindrische Formen, wie sie in meinem Tumor uberwiegen, finde ich kaum. Zweitens sind die Hohlräume weniger regelmässig rund, als buchtig, meist grösser, oft sebr lang gestreckt. In jedem Gesichtsfeld finden sich neben mehr oder weniger grossen Hohlringen zahlreiche, solide Epithelnester, während in meinem Tumor ein mehr einbeitlicherer Bau 
ist, insofern immer grössere Bezirke vorwiegend Hohlringe oder vorwiegend solide Epithelbalken aufweisen. Eine wirkliche Bildung langer, solider Epithelstränge, wie sie die Corticalsubstanz der normalen Thyreoidea und mein Tumor an vielen Stellen aufweist, ist nicht zu erkennen. Das Bindegewebe ist wesentlich zahlreicher, in mehr oder weniger breiten Streifen das Gewebe in Läppchen theilend, auch zwischen den einzelnen Hohlringen finden sich nicht etwa $\mathrm{Ca}$ pillaren, sondern breitere Bindegewebszlige. Dieselben sind zum grössten Theil homogen, nicht faserig, sie färben sich mit den Farbstoffen ähnlich wie das noch zu erwähnende Colloid in den Hohlräumen, bei Färbung nach v. Gieson nicht roth, sondern gelblich, sie enthalten sehr wenige Kerne. Grössere Gefässe finden sich nur in geringer Anzahl, in ibnen aber gelegentlich colloidähnliche Massen, und zwar lediglich in solchen, die sich durch die $A b w e s e n h e i t$ rother Blutzellen und die einfache Wandstructur als Lymphgefässe documentiren. Einige Gefässe enthalten eine mehr feinkörnige Masse, die sich gleich wie das Colloid färbt, von Letzterem jedoch, als einer im Ganzen homogenen Masse, unterschieden ist. Es wird sich wobl um geronnenes Serum handeln.

Das Colloid der Hohlringe hat im Allgemeinen dieselbe Form, wie das unseres Tumors. Doch sind die Contouren durchgehends zackiger, eine bienenwabenähnliche Structur findet sich öfter, die Retraction von dem Epithelring ist stärker, zuweilen baben sich die Epithelien als Randbekleidung der Scholle von dem bindegewebigen Stroma mit der Scholle zusammen retrahirt.

Die Farbennuancen sind bei Färbung mit Hämatoxylin tiefer blau, als bei unserem Tumor, oft ist das Centrum der Scholle metachromatisch grỉn, die zackige Peripherie blau gefärbt. Diese peripheren Theile scheinen z. Th. durch partielle Auflösung der Substanz durchsichtiger und mit Lücken versehen. Mit Hämatoxylin-Eosin färben sich die Schollen mehr oder weniger tiefroth, mit Alauncarmin schmutzigbraunroth, bei Weigert'scher Fibrinfärbung meist nur in den centralen Theilen blau, bei Färbung nach v. Gies on schmutziggriin, viel dunkler als die unseres Tumors.

Der springende Unterschied von unserem Tumor liegt bei dem Jäger'schen also: 1. Im Ueberwiegen des Bindegewebes. 2. In der durchweg cubischen Gestalt der Epithelien. 3. In der meistens satteren Colorirung des Colloids. 4. In dem Ueberwiegen von theils soliden Epithelnestern, theils kleinen Hohlräumen.

In letzterer Hinsicht macht der Jäger'sche Tumor an vielen Stellen direct den Eindruck eines Carcinoms, etwa eines Mamma- 
364 XIX. GoebeL, Ueber eine Geschwulst von schilddrüsenartigem Bau im Femur. .

carcinoms. Auch finden sich im Bindegewebe recht oft kleine Haufen Epithelien, genau wie bei einem Scirrhus. Doch muss auch hier betont werden, dass einerseits tiberall, auch inmitten durehweg solider Zellbaufen die Tendenz zur Hohlraum- und Schollenbildung zu erkennen ist, und eine kleinzellige Infiltration absolut fehlt.

Es sei mir gestattet, meinem hochverehrten Chef, Herrn Geheimrath Helferich, für die guitige Ueberlassung des Materials zu dieser Mittheilung, Herrn Prof. Grawitz für die freundliche Durchsicht der Präparate von Neuem zu danken. 